PAPER

\title{
Childhood onset generalised dystonia can be modelled by increased gain in the indirect basal ganglia pathway
}

\section{T D Sanger}

\author{
Correspondence to: \\ Dr Terence D Sanger, \\ Department of Neurology \\ and Neurosciences, \\ Stanford University \\ Medical Center, 300 \\ Pasteur Drive, A347, \\ Stanford, California \\ 95305-5235, USA; \\ sanger@stanford.edu \\ Received \\ 20 November 2002 \\ In revised form \\ 27 February 2003 \\ Accepted 2 March 2003
}

\begin{abstract}
Clinical experience suggests an important role of the indirect basal ganglia pathway in the genesis of childhood onset generalised dystonia, but it has been difficult to reconcile the increased muscle activity in dystonia with the current model of basal ganglia function in which the indirect pathway is considered primarily inhibitory. The aim of this study was to present a modification of the direct-indirect pathway model, in which the indirect pathway is inverting rather than purely inhibitory, so that while high signals are inhibited, low signals are amplified. As the basal ganglia may be a feedback loop that modifies cortical activity, instability from excessive gain in this feedback loop could explain features of dystonia. A detailed mathematical model is provided, together with simulations of cortical cell population spiking behaviour when connected through a basal ganglia loop. The simulations show that increased gain in the indirect pathway relative to the direct pathway can lead to unstable uncontrolled synchronous oscillations in cortex and basal ganglia. This behaviour could result in dystonia. The model provides a consistent explanation for the association of dystonia with parkinsonism and disorders characterised by dopamine depletion, the ability to treat some dystonias with dopamine, the ability of neuroleptic drug treatment to cause an acute dystonic reaction treatable with anticholinergic drugs, and the ability of pallidotomy or deep brain stimulation of the internal pallidum to alleviate symptoms of generalised dystonia.
\end{abstract}

0 eneralised dystonia is characterised by sustained or intermittent involuntary muscle contractions causing twisting or repetitive movements or abnormal postures. ${ }^{12}$ As dystonia always involves at least some component of excess muscle activity that may lead to excess movement, it is often classified as a hyperkinetic disorder. ${ }^{3}$ However, childhood onset generalised dystonia is associated clinically with diseases such as dopa responsive dystonia ${ }^{4-7}$ and juvenile Parkinson's disease, in which movements may be slow or have reduced amplitude. The reduction of dopaminergic neurones found in Parkinson's disease can also be seen in some forms of primary dystonia, ${ }^{8}$ and an animal model of parkinsonism induced by methyl-phenyl-tetrahydropyridine (MPTP) leads to features of both dystonia and parkinsonism. ${ }^{9}$ It is therefore difficult to reconcile the hyperkinetic features of dystonia with aetiologies that also cause hypokinetic symptoms. ${ }^{10}$

Current models of basal ganglia physiology divide the cortex-basal ganglia-cortex loop into the "direct" and "indirect" pathways. ${ }^{1-13}$ Although there is controversy over this model and it is certainly incomplete, the model has been effective at explaining features of Parkinson's disease and Huntington's disease. The indirect pathway from cortex to striatum to external pallidum to internal pallidum (perhaps through the subthalamic nucleus) to thalamus and back to cortex has an odd number of GABAergic inhibitory synapses and is thus classically thought to be inhibitory. ${ }^{11}{ }^{14-16}$ Deficiency of this pathway is believed to account for the chorea in Huntington's disease. Neuroleptic drugs are thought to increase indirect pathway activity and thus inhibit the excess movements in some choreas.

However, the prevailing model of the indirect pathway as inhibitory fails to explain why D2 specific neuroleptic drugs such as haloperidol, which presumably increase indirect pathway activity, are able to cause dystonia or acute dystonic reactions, nor why more Dl selective neuroleptics such as clozapine do not. ${ }^{17}$ The prevailing model also fails to explain why a selective D2 agonist that presumably decreases indirect pathway activity can relieve dystonic symptoms induced by Dl specific antagonists, ${ }^{18}$ or why dystonia can be associated with a decrease in D2 receptor number ${ }^{19}$ or binding. ${ }^{90}$ Nor can it explain why dopamine, dopamine agonists, or trihexyphenidyl can improve symptoms in several dystonias including the Westphal variant of Huntington's disease, ${ }^{16182122}$ as these drugs would be expected to cause a relative suppression of the indirect pathway. In general, dystonia seems to be associated with decreased striatal dopamine (which would be expected to disinhibit the indirect pathway) and improves with increases in dopaminergic tone (which would be expected to inhibit the indirect pathway). ${ }^{23}$ The preponderance of evidence seems to indicate an association of dystonia with increased activity in the indirect pathway.

Several possible explanations have been proposed to account for the apparent discrepancy between the model of the indirect pathway as inhibitory and the clinical observation of dystonia associated with increased activity in this pathway. Dystonia could result from direct pathway dysfunction, ${ }^{24} 25$ perhaps because of compensation for dopaminergic dysfunction. ${ }^{16}$ It could also reflect combined direct and indirect pathway overactivity. ${ }^{325-27}$ If thalamocortical connections can be inhibitory, then the indirect pathway might have a net excitatory effect. ${ }^{28}$ Thalamostriatal inputs could modify striatal activity. ${ }^{25}$ Enlarged sensory receptive fields might be mediated by indirect pathway activity through the subthalamic nucleus. ${ }^{25}$ If the normal function of the basal ganglia includes a focusing operation with amplification of desired movements and suppression of related but undesired movements ("centre-surround model"), ${ }^{14} 29$ then abnormalities of these patterns could lead to overflow of unwanted movements and inadequate facilitation of desired movements. In particular, dystonia might reflect decreased indirect pathway activity, causing inadequate surround inhibition for muscles not involved in a movement. ${ }^{23}$

In this paper, I propose that the relation between dystonia and function in the indirect pathway can be explained most 
simply by postulating that the odd number of GABAergic inhibitory synapses in the classic model of the indirect pathway is inverting, rather than purely inhibitory. This means that while initially high cortical activity will lead to inhibition after passage around the indirect loop, initially low cortical activity will lead to disinhibition, or relative excitation. The indirect pathway could become unstable if the (negative) gain is excessive, and this would lead to rapid high amplitude uncontrollable oscillations.

\section{METHODS}

In order to demonstrate that excess indirect pathway activity could lead to instability, I will use a simplified model of basal ganglia function focused on the activity of the cortical cells that are the inputs and outputs of the basal ganglia. I will assume that the VA/VL thalamocortical outputs directly affect the inputs to striatum, so that the basal ganglia become part of a closed feedback loop.

I will make use of a probabilistic model of spiking neurones. $^{3031}$ At time $t$, each cortical neurone has a probability of firing $x(t)$ (between 0 and 1 ), and $s_{x}(t)$ is the number of times that the neurone actually fired in the interval $(\mathrm{t}, \mathrm{t}+\Delta \mathrm{t})$ (for short intervals, $\mathrm{s}_{\mathrm{x}}(\mathrm{t})$ will be either 0 or $1)$. The connectivity between neurones is modelled using a common neural network in which the probability of an output neurone firing is a sigmoid function of a linear combination of the spike pattern of the input neurones. For example, if a set of neurones with probability of firing $y_{j}$ connects to a single neurone with probability of firing $\mathrm{x}$, and if $s_{j}$ is the pattern of actual spikes over the input neurones, then $\mathrm{x}=\sigma\left(\sum \mathrm{W}_{\mathrm{j}} \mathrm{s}_{\mathrm{j}}\right)$, where $\mathrm{W}_{\mathrm{j}}$ is the "synaptic weight" connecting input neurone $y_{j}$ to output neurone $x$, and the function $\sigma()$ takes values between 0 and 1 and describes the saturation of firing probability (and spike rate) for large inputs. We do not model refractory periods, but as the simulations will be done using discrete time with a maximum number of spikes per time interval of 1 , effectively there is a refractory period equal to the width of the time bins. This is a very general model of neural behaviour, but other similar models would be expected to show similar behaviour in the simulations below.

Let $\mathrm{x}$ be a vector representing the firing probability of cortical inputs to the striatum, and let $s_{x}$ be a vector of elements that are either 0 or 1 , indicating whether each cell actually fired. Note that we can take the expected value to obtain $E\left[s_{x}\right]=x$. (For low spike rates, this is equivalent to assuming that spikes obey a Poisson distribution with saturating rate $\mathrm{x}$.) Let $\mathrm{z}$ be a vector representing the firing probability of the cortical outputs from the basal gangliathalamocortical circuit. Let A be a matrix for which each entry $a_{i j}$ represents the synaptic connectivity between input neurone $x_{j}$ and output neurone $z_{i}$. Then we can write the linearised approximation to the probability of output firing as $\mathrm{z}=\mathrm{As}_{\mathrm{X}}$ and on average we can take expected values to obtain $\mathrm{a}_{\mathrm{ij}}=\mathrm{E}\left[\partial \mathrm{z}_{\mathrm{i}} / \partial \mathrm{x}_{\mathrm{j}}\right]$, which means that each entry of the matrix A indicates the average change in output neurone probability of firing that is caused by a change in input neurone probability of firing.

If $\mathrm{a}_{\mathrm{ij}}>0$, then an increase in the input firing probability for neurone $x_{j}$ leads to a proportionate increase in the output firing probability for neurone $z_{\mathrm{i}}$, and a decrease in the input firing probability leads to a decrease in the output firing probability. If $\mathrm{a}_{\mathrm{ij}}<0$, then an increase in the input leads to a decrease in the output, and a decrease in the input leads to an increase in the output (inversion). According to the current basal ganglia model, connections for which $\mathrm{a}_{\mathrm{ij}}>0$ will be carried primarily by the direct pathway, while connections for which $\mathrm{a}_{\mathrm{ij}}<0$ will be carried primarily by the indirect pathway. In fact, the effect on output neurones may be the result of a combination of direct and indirect pathway influences, but the sign of each $\mathrm{a}_{\mathrm{ij}}$ will be determined by which pathway dominates that particular connection.

The matrix A describes the overall linearised behaviour of the basal ganglia, and this is a very general approximate model. For example, a focusing or sharpening operation would correspond to a matrix implementing a high pass filter. In the simplest cases, such a matrix would have rows each of which appears to be a "centre-surround" receptive field. The matrix A could also be used to transform one pattern of activity into another, and such a function would allow the basal ganglia to generate successive steps in a movement sequence.

We can separate the matrix A into two matrices, $\mathrm{A}^{+}$and $A^{-}$, with all positive entries, where $A=A^{+}-A^{-}$and $A^{+}$ contains all the direct pathway components while $\mathrm{A}^{-}$ contains the negative of all the indirect pathway components. This decomposition is non-unique, as each element $a_{i j}$ of the original matrix A can be formed from many differences of $\mathrm{a}^{+}{ }_{\mathrm{ij}}$ and $\mathrm{a}^{-}{ }_{\mathrm{ij}}$. If loss of dopaminergic activity leads to decreased gain in the direct pathway (through Dl-like receptors on medium spiny neurones) and increased gain in the indirect pathway (through D2-like receptors on medium spiny neurones), ${ }^{11}{ }^{32-34}$ then the components of $\mathrm{A}$ in the direct pathway will decrease, while components in the indirect pathway will increase. (There are certainly other effects of dopamine, including modulation of large aspiny cholinergic interneurones, but such effects are not included in the classic model and will be ignored here.) This leads to a modified $\mathrm{A}^{\prime}$, and we can write: $\mathrm{A}^{\prime}=\mathrm{pA}^{+}-\mathrm{qA}^{-}$where $\mathrm{p}<\mathrm{l}$ and $\mathrm{q}>\mathrm{l}$ ( similarly, increased chronic dopaminergic tone would lead to a modified $\mathrm{A}^{\prime}$ with $\mathrm{p}>\mathrm{l}$ and $\mathrm{q}<1$, thereby accentuating the direct pathway); $p$ describes the change in gain in the direct pathway, and $\mathrm{q}$ describes the change in gain in the indirect pathway.

We now assume that the outputs of the basal ganglia circuit directly influence the inputs, so that the circuit forms a closed loop. If $x(t)$ is the probability of firing of cortical cells at time $t$, and $\operatorname{sx}(\mathrm{t})$ is the vector of 0 or 1 elements indicating which cells actually fired, then we have the linearised approximation: $\mathrm{x}(\mathrm{t}+\mathrm{l})=\mathrm{As}_{\mathrm{x}}(\mathrm{t})+\mathrm{Bs}_{\mathrm{u}}(\mathrm{t})$, where $\mathrm{s}_{\mathrm{u}}(\mathrm{t})$ is a set of external inputs to the cortex with firing probability $u(t)$, and the elements $b_{j k}=E\left[\partial x_{j} / \partial u_{k}\right]$ of B specify the influence of each input $\mathrm{u}_{\mathrm{k}}$ on cell $\mathrm{x}_{\mathrm{j}}$. The inputs $\mathrm{u}(\mathrm{t})$ account for sensory, reflex, and voluntary contributions to the motor command. If we take expected values, we obtain: $\mathrm{E}[\mathrm{x}(\mathrm{t}+\mathrm{l})]=\mathrm{Ax}(\mathrm{t})+\mathrm{Bu}(\mathrm{t})$, and we can then treat the actual output $x(t+1)$ as the sum of its expected value plus Poisson-distributed noise:

$$
\mathrm{x}(\mathrm{t}+\mathrm{l})=\mathrm{Ax}(\mathrm{t})+\mathrm{Bu}(\mathrm{t})+\text { noise }
$$

If $\mathrm{A}$ is a symmetrical matrix, then stability will be governed by the magnitude of the principal eigenvalue $\left|\lambda_{\max }\right|$ of A. If $\lambda_{\max }$ is $>1$ then the cortical activity will increase to the maximal firing rate available. If $\lambda_{\max }$ is $<-1$ then the cortical activity will oscillate between the maximum and minimum firing rates available. (For non-symmetrical A, the singular value with largest magnitude will determine stability.) If $\mathrm{A}^{\prime}=\mathrm{pA}^{+}-\mathrm{qA}^{-}$is the modified matrix in response to a change in dopaminergic drive, then increased direct pathway activity will increase $\mathrm{p}$ and increase $\lambda_{\max }$, while increased indirect pathway activity will increase $\mathrm{q}$ and decrease $\lambda_{\max }$. If $\mathrm{p}$ increases sufficiently, then $\lambda_{\max }$ is $>1$, while if $\mathrm{q}$ increases sufficiently, then $\lambda_{\max }$ is $<-1$.

In order to study the effect of surgical lesion or deep brain stimulation (DBS) of the internal globus pallidus, we assume that both treatments result in decreased signal outflow from the basal ganglia. (Similar arguments can be applied to surgical lesions of the thalamus.) In addition to decreasing 
the tonic inhibitory drive to the thalamus, the ability to increase or decrease total output firing in response to cortical input will be reduced owing to the smaller number of output cells. So the decreased outflow dampens both increases and decreases in inhibitory output drive to the thalamus, and we model the effect by an output gain factor $\mathrm{g}$ and a constant $\mathrm{k}$ such that:

$$
\mathrm{E}[\mathrm{x}(\mathrm{t}+\mathrm{l})]=\mathrm{gAx}(\mathrm{t})+\mathrm{Bu}(\mathrm{t})+\mathrm{k}
$$

In the non-surgical condition, $g=1$. Pallidotomy or DBS would be expected to decrease the gain so that $\mathrm{g}$ is $<\mathrm{l}$; g can be thought of as representing the modifiable gain in the internal pallidum (and thalamus).

Surgery would also lead to tonic disinhibition of thalamus and thereby cortex, as represented by the constant term $\mathrm{k}$. It is likely that, over time, the thalamus or cortex would adapt to the reduced inhibition and renormalise the baseline firing rate. However, even if this did not occur, the effect of $\mathrm{k}$ on cortical activity after cycling through the basal ganglia $\mathrm{n}$ times is given by $\mathrm{k}\left(\sum \mathrm{g}^{\mathrm{n}} \mathrm{A}^{\mathrm{n}}\right)$, the stability of which is again determined by the maximum eigenvalue of $\mathrm{A}$ (which is unaffected by k). Therefore $\mathrm{k}$ does not affect whether or not the loop is stable, and we will ignore it in the simulations and discussion.

\section{Simulation}

For the simulation, there are 200 cortical inputs to the basal ganglia circuit, and the output feeds back directly onto these inputs. The $\operatorname{erf}()$ function (Matlab 6.0, Mathworks Inc) is used for the non-linearity $\sigma()$ so that probabilities remain between 0 and 1 . The matrix $\mathrm{A}$ is chosen to be a high pass filter that implements a difference of Gaussian impulse response function that is the one dimensional equivalent of a centre-surround receptive field (centre standard deviation $=2.5$ cells, surround standard deviation $=10$ cells). A is therefore a symmetrical Toeplitz matrix with each row a shifted version of the row above. $\mathrm{A}^{+}$is the positive "centre" Gaussian, and $\mathrm{A}^{-}$is the negative "surround" Gaussian. A broad Gaussian window with a standard deviation of 50 cells was multiplied by the columns of A to reduce artefact from convolution with cells near the edge of the cortical representation. The form of matrix A was chosen based on the suggestion that one function of basal ganglia may be a "focused selection and inhibition" mediated by centresurround-like receptive fields. ${ }^{14}$ However, the instability to be demonstrated would occur with any other choice of matrix for sufficiently large values of q.

The input $\mathrm{Bu}(\mathrm{t}$ ) is a Gaussian excitation (half width $=20$ cells) that represents the command to activate a desired set of cortical cells. It is assumed that this excitation may be inaccurate or noisy, and therefore a fixed noise vector $\mathrm{n}$ with elements $n_{j}$, each chosen independently from a Gaussian distribution with standard deviation 0.1 , is added to the excitation. The vector $\mathrm{n}$ does not change during the experiment. A constant baseline average firing probability of $x^{0}=0.2$ is added to all cortical cells at each time point. Thus the expected probability of firing of the vector of cortical neurones at time $\mathrm{t}+\mathrm{l}$ is given by:

$$
\mathrm{E}[\mathrm{x}(\mathrm{t}+\mathrm{l})]=\mathrm{g}\left(\mathrm{pA}^{+}-\mathrm{qA}^{-}\right) \mathrm{x}(\mathrm{t})+(\mathrm{Bu}(\mathrm{t})+\mathrm{n})+\mathrm{x}^{0}
$$

This update equation is iterated for 150 time points. For the first 100 time points, the input excitation $\mathrm{Bu}(\mathrm{t})+\mathrm{n}$ is on, and for the last 50 time points the excitation is turned off so that any persistent cortical activity can be observed. For the first 50 time points the basal ganglia circuit is disconnected so that the baseline cortical response to $\mathrm{Bu}(\mathrm{t})$ can be observed, and for the last 100 time points the basal ganglia circuit is connected. The values of $\mathrm{p}$ and $\mathrm{q}$ specifying the relative strength of the direct and indirect pathways are varied. The value of $g$ specifying a reduction in overall output (due to surgical lesion or deep brain stimulation of the internal pallidum) is varied.

In a second simulation to study the effect of varying $\mathrm{p}$ and q systematically, random matrices A were created by choosing $\mathrm{A}^{+}$and $\mathrm{A}^{-}$to be matrices of uniformly distributed random entries between 0 and 1 , normalised to have maximum eigenvalue magnitude of 1 . The maximum eigenvalue of $\mathrm{pA}^{+}-\mathrm{qA}^{-}$is averaged for five random matrices, and the result plotted as a function of $\mathrm{p}$ and $\mathrm{q}$.

All simulations were done using Matlab version 6.0 (Mathworks Inc) and the simulation source code can be downloaded from <http://www.stanford.edu/ sanger/ indirect.zip $>$.

\section{RESULTS}

Figure 1 shows simulation results for $p=2, q=2, g=1$. For these values, the maximum eigenvalue of the matrix $\mathrm{A}$ is 0.751 , and therefore the iteration is predicted to be stable. The input excitation $\mathrm{Bu}+\mathrm{n}$ is present alone for the first 50 time points, and this excites a localised set of cells in the middle of the simulated cortex. For the next 50 time points, the input excitation continues, but simulated basal ganglia feedback is added. The result of the feedback is a slight amplification of the stimulated region with relative inhibition in the immediately surrounding regions, and only minor effects in more distant areas of cortex. For the final 50 time points, the input excitation is removed, the feedback continues, and the cortical activity pattern returns toward baseline. This overall pattern would be expected for the normal function of the motor system, in which the basal ganglia excite desired movements and inhibit similar but undesired ones. There should not be persistent excess activity of the cortical pattern after the voluntary input command $\mathrm{Bu}$ stops.

Figure 2 shows simulation results when the strength of the indirect pathway is increased to $\mathrm{q}=8$. In this case, the maximum eigenvalue of $\mathrm{A}$ is $(-) 3.06$, and the iteration is predicted to be unstable. The behaviour for the first 50 time points does not change before the basal ganglia feedback is added. When the feedback is added, however, a rapid oscillation develops which spreads to involve most of the cortical cells. This oscillation continues for some time after the input excitation is removed. This overall pattern represents instability in which there is both spatial overflow to uninvolved areas of cortex (presumably controlling unrelated muscles and muscle groups) and temporal overflow with persistent activity of cortex after removal of the voluntary input command $\mathrm{Bu}$. The pattern of activity shows synchronous oscillations across many of the cortical cells.

Figure 3 shows the simulation with $\mathrm{p}=2$ and $\mathrm{q}=8$, as in fig 2, but the effect of pallidotomy or deep brain stimulation is simulated by setting the overall gain $g=0.2$. This effectively rescues the system from instability (the maximum eigenvalue is $(-) 0.611)$ although the focusing effect expected in the normal case (compare with fig 1) is not as striking.

Figure 4 shows the change in the principal eigenvalue $\left(\lambda_{\max }\right)$ as a function of changes in $p$ and $q$. From the figure, it is clear that stability holds $(-1<$ gain $<1)$ only when $p$ and $q$ have similar values. If $\mathrm{p}>>\mathrm{q}$ then there is instability with gain $>1$, and if $\mathrm{p}<<\mathrm{q}$ there is instability with gain $<-1$. If instability exists because $\mathrm{q}$ is increased, then it can be relieved by a compensatory increase in p. Similarly, if instability exists because $\mathrm{p}$ is decreased below $\mathrm{q}$, then it can be relieved by a compensatory decrease in q. 

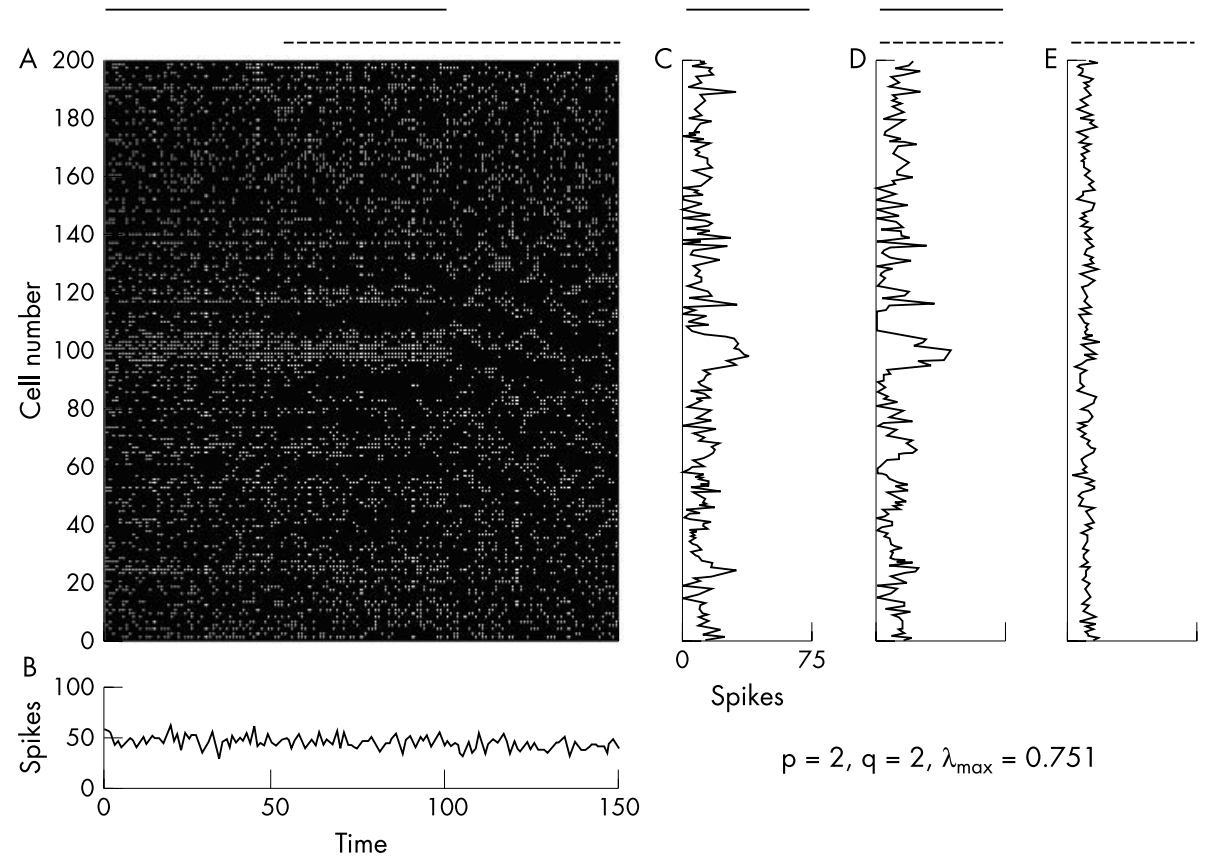

$p=2, q=2, \lambda_{\text {max }}=0.751$

Figure 1 Model of normal behaviour of the system. Simulation parameters are shown, along with the resulting magnitude of the maximum eigenvalue. (A) Spike raster for 200 cells simulated simultaneously over 150 time points. Time increases from left to right. The solid line above the figure indicates the duration of the input stimulus Bu+n. The dashed line indicates when the basal ganglia circuit is active. (B) The total number of spikes present at each time point summed over the full population of cells. (C) The total number of spikes for each cell, summed over the first 50 time points (basal ganglia inactive). (D) The total number of spikes for each cell, summed over the middle 50 time points (basal ganglia active). (E) The total number of spikes for each cell, summed over the final 50 time points (basal ganglia active, but input stimulus removed).

\section{DISCUSSION}

The results show that increased gain in the indirect pathway can lead to instability in the basal ganglia loop, with consequent spread of activity and persistent involuntary activity. This finding is predicted by modifying the prevailing model of basal ganglia function, ${ }^{11-13}$ so that the indirect
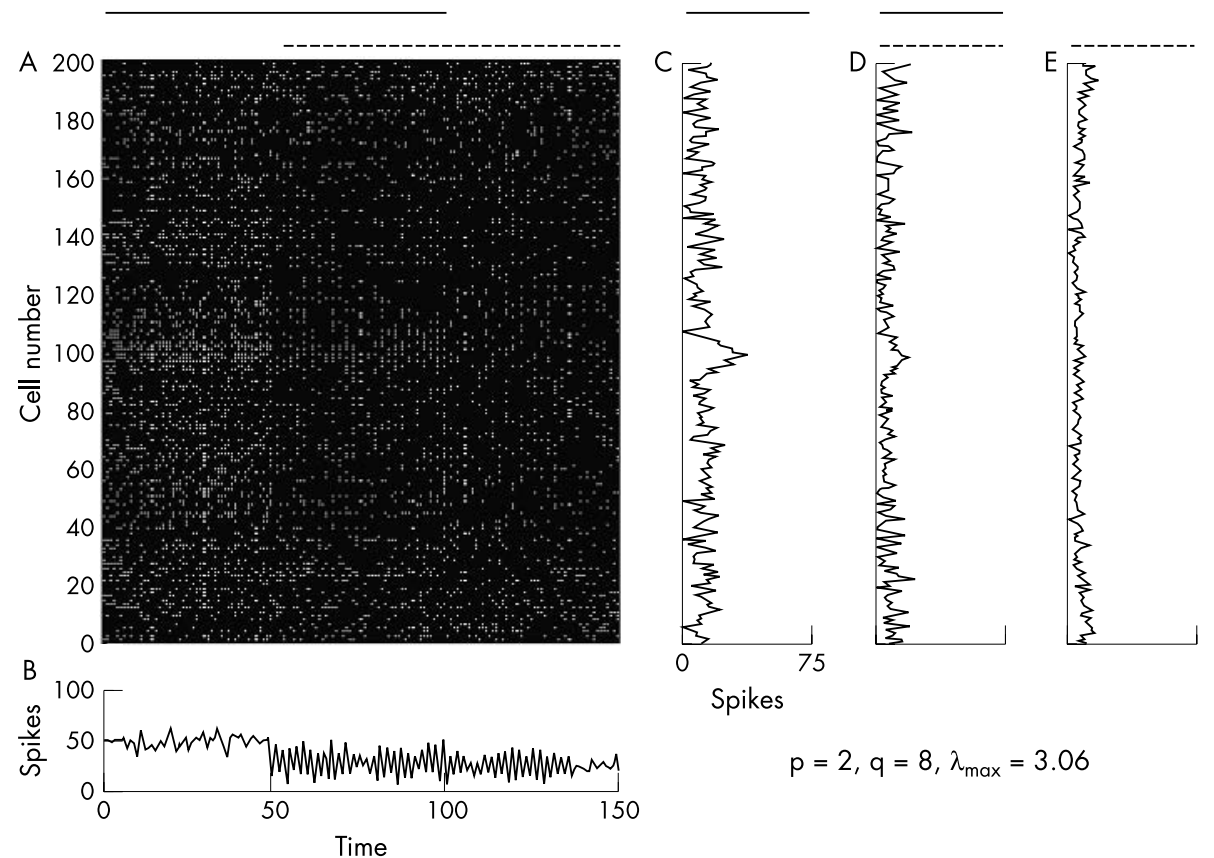

Figure 2 Model of dystonic behaviour. (A) Spike raster for 200 cells simulated simultaneously over 150 time points. Time increases from left to right. The solid line above the figure indicates the duration of the input stimulus Bu+n. The dashed line indicates when the basal ganglia circuit is active. (B) The total number of spikes present at each time point summed over the full population of cells. Note the rapid oscillation that occurs over the full population of cells. (C) The total number of spikes for each cell, summed over the first 50 time points (basal ganglia inactive). (D) The total number of spikes for each cell, summed over the middle 50 time points (basal ganglia active). (E) The total number of spikes for each cell, summed over the final 50 time points (basal ganglia active, but input stimulus removed). 


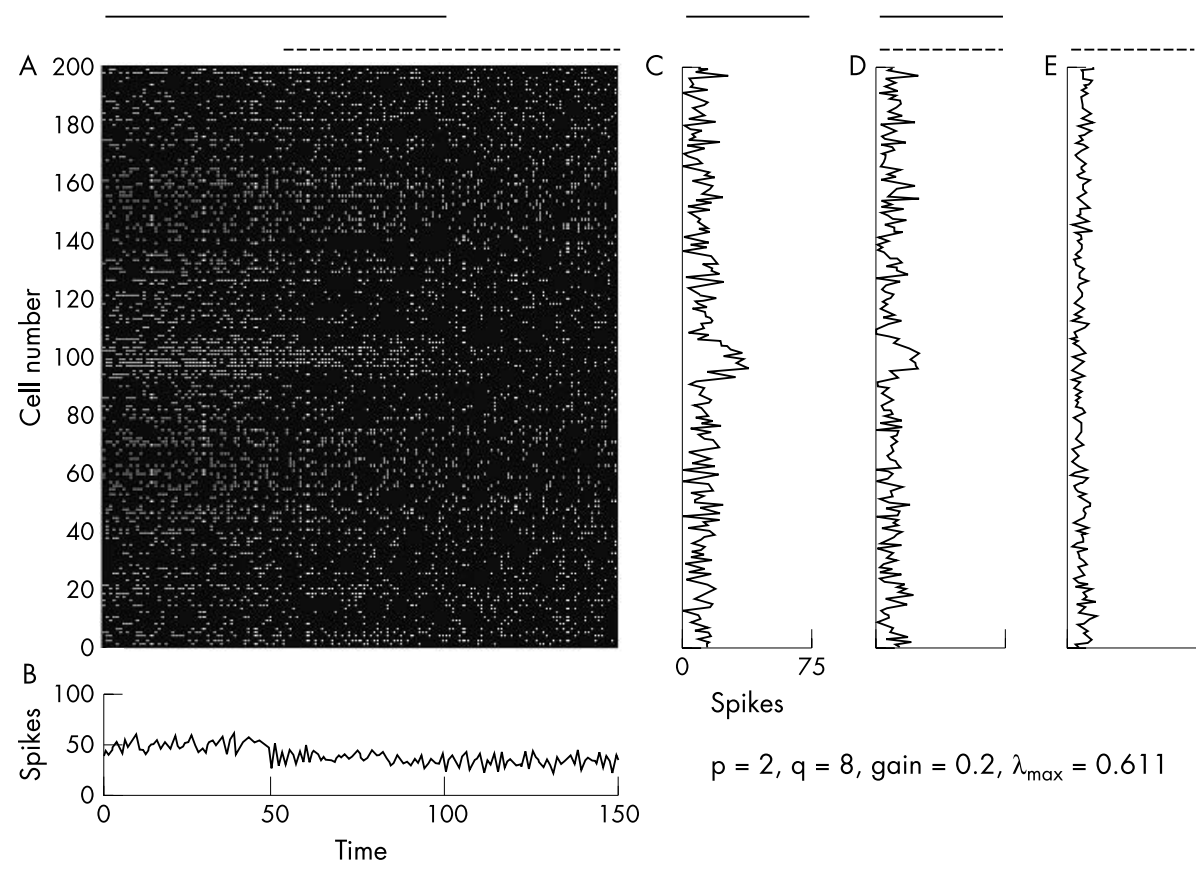

Figure 3 Model of rescue of dystonia by pallidotomy or deep brain stimulation of internal pallidum. (A) Spike raster for 200 cells simulated simultaneously over 150 time points. Time increases from left to right. The solid line above the figure indicates the duration of the input stimulus Bu+n. The dashed line indicates when the basal ganglia circuit is active. (B) The total number of spikes present at each time point summed over the full population of cells. (C) The total number of spikes for each cell, summed over the first 50 time points (basal ganglia inactive). (D) The total number of spikes for each cell, summed over the middle 50 time points (basal ganglia active). (E) The total number of spikes for each cell, summed over the final 50 time points (basal ganglia active, but input stimulus removed). Parameters are the same as in fig 2, but the overall gain of the basal ganglia loop is decreased by a factor of $5(\mathrm{~g}=0.2)$.

pathway is inverting, rather than purely inhibitory. With this added assumption, the model is sufficient to explain instability caused by excess indirect pathway gain. The mathematical features of the model are summarised in fig 5 , and if all other gains are equal, then the overall gain can be conceptualised by the relation: Gain $=g(p-q)$, where $g$ is the combined output (pallidal+thalamic) gain, $p$ is the gain in the direct pathway, and $\mathrm{q}$ is the gain in the indirect pathway. In this model, dystonia occurs for movements where gain is $<-1$, bradykinesia occurs when $0>$ gain $>-1$, and dyskinesia or chorea occur when gain is $>1$.

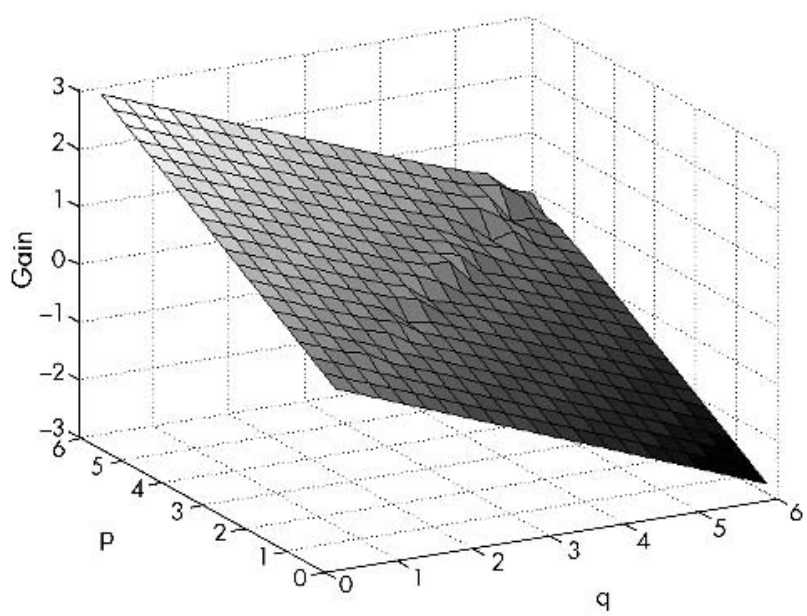

Figure 4 Calculation of gain as a function of $\operatorname{direct}(p)$ and indirect ( $q$ ) pathway gains for random matrices. Each value is the average gain for five different random matrices. The basal ganglia loop is predicted to be stable only for values of gain between -1 and 1 .
Note that this model can remain consistent with Mink's hypothesis for basal ganglia function of "pattern selection and inhibition" ${ }^{\prime 14}$ if selection and inhibition are considered to

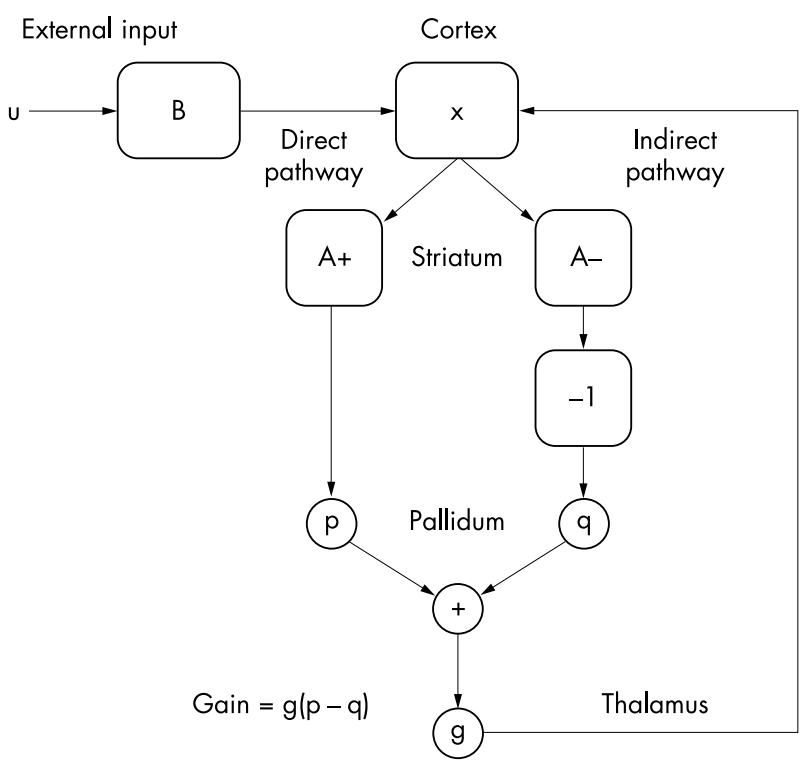

Figure 5 Illustration of the model. External input $u$ excites the cortex $x$. Cortical activity is sent through the $\operatorname{direct}(\mathrm{A}+)$ and indirect $(\mathrm{A}-$ ) pathways. The indirect pathway has a " -1 " term to indicate that its overall effect is inverting. The two pathways are recombined in the internal pallidum and returned back via thalamus to the cortex $x$ (this model ignores brain stem outputs through the substantia nigra pars reticulata). The overall gain in the loop is controlled by the direct pathway gain $p$, the indirect pathway gain $q$, and the pallido-thalamic output gain $g$, and if all other gains are 1 then it is equal to $g(p-q)$. 
apply to combined patterns of cortical excitation and inhibition. By inverting the cortical pattern, the indirect pathway in the model proposed here is de-emphasising both the increased and decreased elements of the cortical pattern, while the direct pathway is emphasising both the increased and decreased elements. The operation could perhaps be thought of as increasing or decreasing the contrast of patterns of cortical activity.

We cannot determine whether instability in this model does in fact describe the pathophysiology of childhood onset generalised dystonia, nor whether there might be other mechanisms that can cause dystonia. Nevertheless, the model is consistent with several findings that have not yet been fully explained. In particular, it explains how a decrease in dopaminergic tone (which increases q), as seen in dopa responsive dystonia, could lead to dystonia. It explains how blockade of D2-like receptors with neuroleptics (which increases q) could lead to dystonia and acute dystonic reactions. As anticholinergic drug treatment would be expected to inhibit indirect pathway gain, this model explains how anticholinergic drugs could suppress dystonia (by decreasing q). Finally, if we assume that a lesion in the output of the basal ganglia can decrease the overall gain in the loop (by decreasing g) and thereby reduce instability, then this model provides a new explanation for the benefit of pallidotomy or deep brain stimulation for dystonic symptoms. ${ }^{162425}$

This model predicts that dystonia may be caused not only by increased indirect pathway gain q, but also in some cases by a decrease in direct pathway gain $\mathrm{p}$, such that the direct pathway fails to compensate for the negative bias induced by the indirect pathway. While it is difficult to relate specific effects of combined drug treatment trials because of the unknown relative potency of different drugs, it has been shown that dystonia can be caused by both specific Dl antagonists (which decrease direct pathway gain $p$ ) and specific D2 antagonists (which increase indirect pathway gain q). ${ }^{35}$ Further, dystonia induced by a Dl antagonist may be rescued by a D2 agonist. ${ }^{35}$

Although it was not my original intent, the model is consistent with features of bradykinesia, dyskinesia in Parkinson's disease, and chorea in Huntington's disease. In particular, bradykinesia might be expected to occur for moderate negative values of gain between 0 and -1 , as values in this range would dampen the cortical pattern of activity without causing instability. Chorea or dyskinesia (and possibly some forms of dystonia) might be expected to occur for large positive values of gain, and this would be a predicted effect of both dopaminergic and anticholinergic drugs at high doses.

The new model may be able to explain features of surgical intervention for Parkinson's disease. In particular, a lesion or DBS in the internal pallidum would cause a decrease in output gain g, which could lessen dyskinesias and thereby allow an increase in dopaminergic drug treatment. A lesion in the subthalamic nucleus would reduce gain only in the indirect pathway q, so it would increase the overall loop gain without the need for drug treatment. The predicted effects of the two surgical procedures are consistent with the clinical observation that pallidotomy or pallidal DBS in Parkinson's disease leads to a reduction in dyskinesia but necessitates continued dopaminergic drug treatment, while subthalamotomy or subthalamic DBS may lead to a reduced need for drug treatment. ${ }^{36} 37$

To understand how dystonic and bradykinetic symptoms could co-exist in children, it is important to realise that dystonia requires instability only of the largest eigenvalue $\lambda_{\max }$. The eigenvalues for other components could be negative (but greater than -1 ), so that a small number of unstable dystonic patterns might exist on a background of other patterns with bradykinesia.

This model does not address the evolution of symptoms over time, the need for prolonged treatment with dopaminergic or anticholinergic drugs, the development of tardive dystonia or chorea, dystonia caused by acquired lesions, the resolution of some types of dystonia with antidopaminergic drugs including tetrabenazine, or the slow improvement in dystonic symptoms following stereotactic surgery. It does not explain the enlarged sensory receptive fields found in the pallidum of adults with generalised dystonia ${ }^{24} 25$ or the abnormal cortical representations in adult onset focal dystonia..$^{28}{ }^{38-41}$ The effect of dopamine on corticostriatal long term potentiation and long term depression may be an important factor in these phenomena, but explanation will need to await future models. This model also does not yet attempt to make specific predictions of average neuronal firing rates. There is evidence that firing in the internal pallidum is reduced in dystonia, ${ }^{25}$ but this effect may partly reflect repeated movement attempts. ${ }^{24}$

There are several important assumptions of this model that have not yet been verified experimentally. Instability in feedback depends upon the outputs of the basal ganglia loop projecting back to the same input cells. If this is not the case, then excess indirect pathway activity could still invert the pattern of cortical activity but it would not lead to feedback instability. It is possible that inversion of the desired cortical activity pattern would be sufficient to explain clinical features of dystonia, but this would be different from the feedback model proposed here.

Another important assumption is that a decrease in cortical activity leads to a decrease in striatal activity. For this to be meaningful, the baseline activity of medium spiny neurones during movement would have to be sufficient for a decrease to be detectable. At rest, the baseline firing rate of these neurones may be very low, ${ }^{42}$ so the model assumes that the rate during activity is higher. As medium spiny neurones can exist in either an "up" (relatively depolarised) or "down" (relatively hyperpolarised) state, ${ }^{43-45}$ this model also requires that at least some fraction of the medium spiny neurones be in the "up" state during movement in order for changes in firing rate to be detectable.

There is little doubt that the direct/indirect pathway model of basal ganglia function will need to be modified as new clinical and basic science information becomes available (see, for example, Parent and Hazrati ${ }^{46}{ }^{47}$ ). Nevertheless, the results here show that by treating the indirect pathway as inverting, the two pathway model is at least sufficient to explain instability caused by decreased dopaminergic tone. Instability in the indirect pathway could be a cause of childhood onset generalised dystonia.

\section{ACKNOWLEDGEMENTS}

I wish to thank Jon Mink for extensive comments and discussion of the manuscript. Part support was provided during this research by grant K23-NS41243 from the NINDS.

\section{Authors' affiliations \\ T D Sanger, Department of Neurology and Neurosciences, Stanford University Medical Center, Stanford, California, USA}

Competing interests: none declared

\section{REFERENCES}

1 Fahn S, Bressman SB, Marsden CD. Classification of dystonia. Adv Neurol 1998;78:1-10.

2 Bressman SB. Dystonia [In-process citation]. Curr Opin Neurol 1998;11:363-72.

3 Vitek JL, Giroux M. Physiology of hypokinetic and hyperkinetic movement disorders: model for dyskinesia. Ann Neurol 2000;47(suppl 1):S131-40. 
4 Segawa $M$, et al. Hereditary progressive dystonia with marked diurnal fluctuation. Adv Neurol 1976;14:215-33.

5 Harwood G, et al. Lessons from a remarkable family with dopa-responsive dystonia. J Neurol Neurosurg Psychiatry 1994;57:460-3.

6 Nishiyama N, et al. Gene mutation in hereditary progressive dystonia with marked diurnal fluctuation (HPD), strictly defined dopa-responsive dystonia. Brain Dev 2000;22(suppl 1):S102-6.

7 Segawa M, Nishiyama N, Nomura Y. DOPA-responsive dystonic parkinsonism - pathophysiologic considerations. Adv Neurol 1999:80:389-400.

8 Playford ED, et al. Striatal $\left[{ }^{18} \mathrm{~F}\right]$ dopa uptake in familial idiopathic dystonia. Brain 1993; 116:1191-9.

9 Perlmutter JS, et al. MPTP induces dystonia and parkinsonism. Clues to the pathophysiology of dystonia. Neurology 1997;49:1432-8.

10 Berardelli A, et al. The pathophysiology of primary dystonia. Brain 1998;121:1195-212.

11 Albin RL, Young AB, Penney JB. The functional anatomy of basal ganglia disorders. Trends Neurosci 1989; 12:366-75.

12 DeLong MR. Primate models of movement disorders of basal ganglia origin. Trends Neurosci 1990;13:281-5.

13 Alexander GE, Crutcher MD. Functional architecture of basal ganglia circuits: neural substrates of parallel processing. Trends Neurosci 1990;13:266-71.

14 Mink JW. The basal ganglia: focused selection and inhibition of competing motor programs. Prog Neurobiol 1996;50:381-425.

15 Brown J, Bullock D, Grossberg S. How the basal ganglia use parallel excitatory and inhibitory learning pathways to selectively respond to unexpected rewarding cues. J Neurosci 1999;19:10502-11.

16 Wichmann T, DeLong MR. Models of basal ganglia function and pathophysiology of movement disorders. Neurosurg Clin North Am 1998;9:223-36.

17 Casey DE. Dopamine D1 (SCH 23390) and D2 (haloperidol) antagonists in drug-naive monkeys. Psychopharmacology 1992;107:18-22.

18 Kistrup K, Gerlach J. Selective D1 and D2 receptor manipulation in Cebus monkeys: relevance for dystonia and dyskinesia in humans. Pharmacol Toxicol 1987;61:157-61.

19 Todd RD, et al. Dynamic changes in striatal dopamine D2 and D3 receptor protein and mRNA in response to 1-methyl-4-phenyl-1,2,3,6tetrahydropyridine (MPTP) denervation in baboons. J Neurosci 1996; 16:7776-82.

20 Naumann $M$, et al. Imaging the pre- and postsynaptic side of striatal dopaminergic synapses in idiopathic cervical dystonia: a SPECT study using [ $\left.{ }^{123} \mid\right]$ epidepride and $\left[{ }^{123} \mid\right]$ beta-CIT. Mov Disord 1998;13:319-23.

21 Bonelli RM, et al. Pramipexole ameliorates neurologic and psychiatric symptoms in a Westphal variant of Huntington's disease. Clin Neuropharmacol 2002;25:58-60.

22 Low PA, Allsop JL, Halmagyi GM. Huntington's chorea: the rigid form (Westphal variant) treated with levodopa. Med J Aust 1974; 1:393-4.

23 Perlmutter J, Mink J. Functional neuroimaging of movement disorders. In: Gilman S, eds. Medlink neurology. San Diego: Medlink Corp, 2001.

24 Lenz FA, et al. Pallidal activity during dystonia: somatosensory reorganisation and changes with severity. J Neurol Neurosurg Psychiatry 1998;65:767-70.

25 Vitek JL, et al. Neuronal activity in the basal ganglia in patients with generalized dystonia and hemiballismus. Ann Neurol 1999;46:22-35.
26 Hallett $M$. Is dystonia a sensory disorder? [editorial]. Ann Neurol 1995;38:139-40.

27 Hallett M. Physiology of basal ganglia disorders: an overview. Can J Neurol Sci 1993:20:177-83.

28 Hallett M. The neurophysiology of dystonia. Arch Neurol 1998;55:601-3.

29 Hikosaka O. Basal ganglia - possible role in motor coordination and learning. Curr Opin Neurobiol 1991;1:638-43.

30 Sanger TD. Probability density estimation for the interpretation of neural population codes. J Neurophysiol 1996;76:2790-3.

31 Sanger TD, Merzenich MM. Computational model of the role of sensory disorganization in focal task-specific dystonia. J Neurophysiol 2000:84:2458-64

32 Alexander GE, Crutcher MD, DeLong MR. Basal ganglia-thalamocortical circuits: parallel substrates for motor, oculomotor, "prefrontal" and "limbic" functions. Prog Brain Res 1990;85: 119-46.

33 Gerfen CR, et al. D1 and D2 dopamine receptor-regulated gene expression of striatonigral and striatopallidal neurons. Science 1990;250:1429-32.

34 Gerfen CR. The neostriatal mosaic: multiple levels of compartmental organization in the basal ganglia. Annu Rev Neurosci 1992;15:285-320.

35 Peacock L, Lublin H, Gerlach J. The effects of dopamine D1 and D2 receptor agonists and antagonists in monkeys withdrawn from long-term neuroleptic treatment. Eur J Pharmacol 1990;186:49-59.

36 Olanow CW, Brin MF, Obeso JA. The role of deep brain stimulation as a surgical treatment for Parkinson's disease. Neurology 2000;55:S60-6.

37 Beijani BP, et al. Deep brain stimulation in Parkinson's disease: opposite effects of stimulation in the pallidum. Mov Disord 1998;13:969-70.

38 Byl NN, Merzenich MM, Jenkins WM. A primate genesis model of focal dystonia and repetitive strain injury. I.Learning-induced dedifferentiation of the representation of the hand in the primary somatosensory cortex in adult monkeys. Neurology 1996;47:508-20.

39 Sanger TD, et al. Nonlinear sensory cortex response to simultaneous tactile stimuli in writer's cramp. Mov Disord 2002;17:105-11.

40 Sanger TD, Tarsy D, Pascual-Leone A. Abnormalities of spatial and temporal sensory discrimination in writer's cramp. Mov Disord 2001;16:94-9.

41 Marsden CD, Rothwell JC. The physiology of idiopathic dystonia. Can J Neurol Sci 1987;14(suppl 3):521-7.

42 Wilson CJ, Groves PM. Spontaneous firing patterns of identified spiny neurons in the rat neostriatum. Brain Res 1981;220:67-80.

43 Wilson $\mathrm{CJ}$. The generation of natural firing patterns in neostriatal neurons. In: Arbuthnott GW, Emson PC, eds. Progress in brain research: chemical signaling in the basal ganglia. Elsevier: Amsterdam, 1993:277-98.

44 Wilson CJ, Kawaguchi Y. The origins of two-state spontaneous membrane potential fluctuations of neostriatal spiny neurons. J Neurosci 1996; 16:2397-410.

45 Stern EA, Kincaid AE, Wilson CJ. Spontaneous subthreshold membrane potential fluctuations and action potential variability of rat corticostriatal and striatal neurons in vivo. J Neurophysiol 1997;77:1697-715.

46 Parent A, Hazrati LN. Functional anatomy of the basal ganglia. I.The corticobasal ganglia-thalamo-cortical loop. Brain Res Brain Res Rev 1995;20:91-127.

47 Parent A, Hazrati LN. Functional anatomy of the basal ganglia. II. The place of subthalamic nucleus and external pallidum in basal ganglia circuitry. Brain Res Brain Res Rev 1995;20:128-54. 